JHU-TIPAC-97023

hep-ph/9712364

December 1997

\title{
Heavy Quark Effective Theory and Inclusive $B$ Decays
}

\author{
Adam F. Falk \\ Department of Physics and Astronomy, The Johns Hopkins University \\ 3400 North Charles Street, Baltimore, Maryland 21218 USA \\ falk@jhu.edu
}

\begin{abstract}
I review the use of the Heavy Quark Effective Theory in the computation of total rates and differential kinematic distributions in inclusive semileptonic and radiative $B$ decays. Particular attention is paid to strategies for the extraction of $V_{c b}$ and $V_{u b}$.
\end{abstract}

To appear in the Proceedings of Beauty '97

University of California, Los Angeles

October 12-16, 1997 


\section{THE HEAVY QUARK EXPANSION FOR INCLUSIVE $B$ DECAYS}

Why is it important to have a good theoretical understanding of inclusive weak $B$ decays? One might be tempted to take the point of view that since the short distance structure of these decays is presumably known, all that is left to do is to sort out some messy but ultimately unenlightening details of the QCD dynamics. However, by doing so one would forget the important fact that while the structure of these decays is indeed well understood, the strengths of the weak decay couplings of the $b$ quark are not yet known with sufficient precision. The weak decay of the $b$ is governed by two fundamental constants which appear as coefficients of currents found in the weak interaction Lagrangian. The larger of the two is $V_{c b}$, which is multiplies the current $\bar{c} \gamma^{\mu}\left(1-\gamma^{5}\right) b$; the smaller is $V_{u b}$, which multiplies $\bar{u} \gamma^{\mu}\left(1-\gamma^{5}\right) b$. Respectively, they govern the rates for the inclusive semileptonic decays $B \rightarrow X_{c} \ell \nu$ and $B \rightarrow X_{u} \ell \nu$. For reasons of theoretical simplicity, I will confine myself to the discussion of semileptonic, rather than nonleptonic, weak $B$ decays. In addition, I will discuss radiative decays of the form $B \rightarrow X_{s, d} \gamma$. These transitions, which are mediated in the Standard Model by one-loop penguin operators, may also be studied with the techniques to be described here.

The theoretical tool which is used to analyse semileptonic $B$ decays is the Operator Product Expansion (OPE) [2, 3]. The OPE exploits a fact known as parton-hadron duality, which is, roughly speaking, the observation that if the energy release in a decay is large $\left(m_{b} \gg \Lambda_{\mathrm{QCD}}\right)$ and the decay is sufficiently inclusive, then it is essentially controlled by physics at short distances. Long distance effects, which arise from the $B$ meson bound state structure, appear only as subleading corrections. The result is an expansion for the semileptonic width $\Gamma$ of the generic form

$$
\Gamma=\Gamma_{0}\left[1+\frac{\Lambda_{1}}{m_{b}}+\frac{\Lambda_{2}^{2}}{m_{b}^{2}}+\ldots\right],
$$

where $\Gamma_{0}$ is the decay rate of a free $b$ quark, and $\Lambda_{i}$ are nonperturbative parameters of order $\Lambda_{\mathrm{QCD}}$ which are independent of $m_{b}$ in the limit $m_{b} \rightarrow \infty$. The quantities $\Lambda_{i}$ cannot be computed theoretically from first principles (except, perhaps eventually, on the lattice); instead, they must be modeled or, preferably, be measured. It is the current state of the art to include terms in $\Gamma$ up to order $1 / m_{b}^{3}$. Those of order $1 / m_{b}$ and $1 / m_{b}^{2}$ are taken seriously, while those of order $1 / m_{b}^{3}$ are used (usually crudely) to estimate the residual uncertainties, which are typically at the level of a few percent.

Simultaneously, one may exploit the fact that the the QCD coupling constant $\alpha_{s}$ is perturbative at the scale $m_{b}$, since $\alpha_{s}\left(m_{b}\right) \simeq 0.2$. Since the typical gluon carries a momentum set by the scale $m_{b} \gg \Lambda_{\mathrm{QCD}}$, the theory is in the region where asymptotic freedom applies. As a result, each term in the expansion (1) is actually a power series in $\alpha_{s}\left(m_{b}\right)$, leading to the refined (schematic) form

\footnotetext{
${ }^{1}$ Actually, the analysis of nonleptonic decays is on a less rigorous theoretical footing than that of semileptonic decays [1].
} 


$$
\Gamma=\Gamma_{0}\left[C_{0}\left(\alpha_{s}\right)+C_{1}\left(\alpha_{s}\right) \frac{\Lambda_{1}}{m_{b}}+C_{2}\left(\alpha_{s}\right) \frac{\Lambda_{2}^{2}}{m_{b}^{2}}+\ldots\right] .
$$

It is the present state of the art to compute $C_{1}$ to order $\alpha_{s}$ and $C_{0}$ to "order" $\alpha_{s}^{2} \beta_{0}$, where $\beta_{0}=9$ is the first coefficient in the QCD beta function. Such terms, while not formally dominant in any nearby limit, are often the largest contribution to the two loop correction 四. Here the largest uncertainties are from the choice of renormalization scale $\mu$, and from infrared renormalons which appear, in principle, at high orders in the perturbative expansion. The renormalons will be discussed further below.

To the order which they are computed, there are four input parameters which appear in the expansion. First, the QCD perturbation series depends on $\alpha_{s}\left(m_{b}\right)$, which we will treat as known, since the uncertainty associated with its value is comparatively small. Second, there are parameters which arise in the OPE. The most important of these is $\bar{\Lambda}$, defined by

$$
\bar{\Lambda}=\lim _{m_{b} \rightarrow \infty}\left[M_{B}-m_{b}^{\text {pole }}\right] .
$$

The constituent quark model, along with QCD sum rule estimates, lead one to expect $200 \mathrm{MeV} \leq \bar{\Lambda} \leq 700 \mathrm{MeV}$. At the next order, there appear two matrix elements of dimension five operators [5],

$$
\begin{aligned}
\lambda_{1} & =\left\langle B\left|\bar{b}(i D)^{2} b\right| B\right\rangle / 2 M_{B}, \\
\lambda_{2} & =\left\langle B\left|\bar{b}\left(-\frac{g}{2} \sigma^{\mu \nu} G_{\mu \nu}\right) b\right| B\right\rangle / 6 M_{B} .
\end{aligned}
$$

The parameter $\lambda_{1}$ is related to the negative of the $b$ quark kinetic energy in the $B$ meson; models and theoretical prejudice would indicate that it lies in the range $0 \leq-\lambda_{1} \leq 1 \mathrm{GeV}^{2}$. The parameter $\lambda_{2}$ is related to the matrix element of the leading operator which violates heavy spin symmetry, and is proportional to the $B-B^{*}$ mass splitting. Neglecting a small radiative correction, $\lambda_{2} \approx 0.12 \mathrm{GeV}^{2}$. In what follows, we will treat $\alpha_{s}$ and $\lambda_{2}$ as known, and $\bar{\Lambda}$ and $\lambda_{1}$ as parameters which must be determined somehow from experiment.

In fact, there is an additional subtlety associated with the definition of $\bar{\Lambda}$, or more precisely, the definition of $m_{b}^{\text {pole }}$. The problem is that $m_{b}^{\text {pole }}$ is not well defined in QCD. Of course, nonperturbatively there is no pole in the fully dressed quark propagator, hence no unambiguous definition of $m_{b}^{\text {pole }}$. But even within QCD perturbation theory, the asymptotic nature of the expansion leads to an ambiguity in the perturbative definition of $m_{b}^{\text {pole }}$. In particular, one can attempt to sum the "BLM-enhanced" terms proportional to $\alpha_{s}^{n} \beta_{0}^{n-1}$, where $\beta_{0}$ is the first coefficient in the QCD beta function, using Borel resummation techniques. However, one finds an obstruction in the Borel plane due to an infrared renormalon ambiguity, which must be resolved by the choice of a resummation scheme. What this means, essentially, is that $m_{b}^{\text {pole }}$ has a scheme dependence in its definition, quite analogous to the renormalization scale dependence of quantities defined in dimensional regularization with $\overline{\mathrm{MS}}$ subtraction. As with the renormalization group, this scheme dependence cancels in physical quantities, if the calculation is organized self-consistently. The problem is not with the predictivity of the theory, but with the physical interpretation of $m_{b}^{\text {pole }}$. Since the renormalon ambiguity is of order $100 \mathrm{MeV}$ [4, 6, 7], there is no "preferred" value for $m_{b}^{\text {pole }}$ within a smaller precision than this. The most important practical point is that if the renormalon ambiguity is not canceled consistently, then there is an irreducible uncertainty in the 
input $\bar{\Lambda}$, which it inherits from $m_{b}^{\text {pole }}$. One of the issues which I will address is the consistent treatment of this effect.

\section{INCLUSIVE SEMILEPTONIC $B$ DECAY}

I turn first to the extraction of $\left|V_{c b}\right|$ from the inclusive decay $B \rightarrow X_{c} \ell \nu$. The expression for the semileptonic width is written most conveniently as a function of the ratio of spin averaged masses $\bar{M}_{D} / \bar{M}_{B}$, where $\bar{M}_{D}=\left(M_{D}+3 M_{D^{*}}\right) / 4$ and $\bar{M}_{B}=\left(M_{B}+3 M_{B^{*}}\right) / 4$. These physical masses are, in turn, functions of the quark masses $m_{b}$ and $m_{c}$ and the hadronic parameters $\bar{\Lambda}, \lambda_{1}$ and $\lambda_{2}$, according to

$$
\begin{gathered}
M_{B}=m_{b}^{\text {pole }}+\bar{\Lambda}-\frac{\lambda_{1}+3 \lambda_{2}}{2 m_{b}}+\ldots \\
M_{B^{*}}=m_{b}^{\text {pole }}+\bar{\Lambda}-\frac{\lambda_{1}-\lambda_{2}}{2 m_{b}}+\ldots
\end{gathered}
$$

The result for the semileptonic width is [3, 8, 9, 10

$$
\begin{aligned}
\Gamma\left(B \rightarrow X_{c} \ell \nu\right)= & \frac{G_{F}^{2}\left|V_{c b}\right|^{2} M_{B}^{5}}{192 \pi^{3}} f\left(\bar{M}_{D} / \bar{M}_{B}\right)\left\{1-1.54 \frac{\alpha_{s}}{\pi}-\left(1.43 \beta_{0}+c\right)\left(\frac{\alpha_{s}}{\pi}\right)^{2}\right. \\
& \left.-1.65 \frac{\bar{\Lambda}}{M_{B}}\left(1-0.87 \frac{\alpha_{s}}{\pi}\right)-0.95 \frac{\bar{\Lambda}^{2}}{M_{B}^{2}}-3.18 \frac{\lambda_{1}}{M_{B}^{2}}+0.02 \frac{\lambda_{2}}{M_{B}^{2}}+\ldots\right\}
\end{aligned}
$$

where $f\left(\bar{M}_{D} / \bar{M}_{B}\right)=0.369$ is a phase space factor, and the two loop coefficient $c$ is known to be small [11]. An important feature of the QCD expansion is that there is also a renormalon ambiguity in the perturbation series in Eq. (6); that is, there is a scheme dependence in the summation of the series of terms proportional to $\alpha_{s}^{n} \beta_{0}^{n-1}$. However, one can show that this ambiguity precisely compensates the renormalon ambiguity in $\bar{\Lambda}$, so the scheme dependence cancels in the physical width [4]. The mechanism is similar to the cancelation of renormalization scale dependence in physical quantities.

Once $\Gamma\left(B \rightarrow X_{c} \ell \nu\right)$ has been measured, the expression (6) can be used to extract $\left|V_{c b}\right|$. There is a variety of sources of uncertainty in this determination. First, both the perturbative QCD expansion and the operator product expansion are truncated, and we must estimate the size of the omitted terms. For the radiative corrections, the leading unknown term is of order $\left(\alpha_{s} / \pi\right)^{2}$, and is expected to be no larger than a few percent. For the nonperturbative corrections, the next terms are of order $\left(\bar{\Lambda} / M_{B}\right)^{3}$, also at the level of a percent or so. Second, there is uncertainty in the values of the parameters to be inserted in the terms which have been calculated. By far the most important of these is the uncertainty in $\bar{\Lambda}$, or equivalently in $m_{b}^{\text {pole }}$, because it enters the expansion already at first order. For example, an uncertainty in $\bar{\Lambda}$ of $200 \mathrm{MeV}$ implies an uncertainty in $\left|V_{c b}\right|$ of approximately $10 \%$. This is the dominant source of error in the determination of $\left|V_{c b}\right|$.

The cleanest and best way to reduce this uncertainty is to determine $\bar{\Lambda}$ directly from the semileptonic decays themselves. This is possible because one can compute not only total decay rates with the OPE, but kinematic distributions as well. While there exist interesting pathologies in certain of these distributions, low moments of kinematic observables are 
almost always well behaved. In particular, it is useful to consider the following two types of quantities:

(i) In the decay $B \rightarrow X_{c} \ell \nu$, let $s_{H}$ be the invariant mass of the hadronic state $X_{c}$. Then the moments of the form $\left\langle\left(s_{H}-\bar{M}_{D}^{2}\right)^{n}\right\rangle$ have an OPE and a perturbative QCD expansion. Constructed in this way, the first moment starts at order $\bar{\Lambda} M_{B}$, while the second starts at order $\bar{\Lambda}^{2}$. Since terms of the order $\bar{\Lambda}^{3}$ are sources of uncertainty, the first moment is substantially more reliable theoretically than is the second. These moments have been studied in detail in Refs. [12,13].

(ii) One may also study the differential distribution $\mathrm{d} \Gamma / \mathrm{d} E_{\ell}$ and its moments $\left\langle E_{\ell}^{n}\right\rangle$. Again, these quantities have an OPE and a perturbative QCD expansion. They have been studied in detail in Refs. 14, 15.

Using a calculation analogous to that of the total semileptonic decay rate, these kinematic moments have expansions in terms of $\alpha_{s}, \bar{\Lambda}, \lambda_{1}$ and $\lambda_{2}$. As with the total rate, the renormalon ambiguity in the perturbative series cancels that in $\bar{\Lambda}$. Thus, if one writes $\Gamma\left(B \rightarrow X_{c} \ell \nu\right)$ in terms of one of these moments, eliminating $\bar{\Lambda}$, the renormalon ambiguity cancels automatically in the resulting expression. For example, if we eliminate $\bar{\Lambda}$ in favor of $s_{1} \equiv\left\langle s_{H}-\bar{M}_{D}^{2}\right\rangle$ and solve for $\left|V_{c b}\right|$, we find

$$
\begin{aligned}
\left|V_{c b}\right|=[ & {\left[\frac{\Gamma\left(B \rightarrow X_{c} \ell \nu\right)}{G_{F}^{2} M_{B}^{5} / 192 \pi^{3}}\right]^{-\frac{1}{2}}\left\{1+0.59 \frac{\alpha_{s}}{\pi}+\left(0.37 \beta_{0}+c^{\prime}\right)\left(\frac{\alpha_{s}}{\pi}\right)^{2}\right.} \\
& \left.\quad+0.68 \frac{s_{1}}{M_{B}^{2}}\left(1+0.70 \frac{\alpha_{s}}{\pi}\right)+0.38 \frac{s_{1}^{2}}{M_{B}^{4}}-2.00 \frac{\lambda_{1}}{M_{B}^{2}}+1.14 \frac{\lambda_{2}}{M_{B}^{2}}+\ldots\right\} .
\end{aligned}
$$

In this expression, the two loop contributions and the uncertainty due to $\lambda_{1}$ are both at the level of one percent. The effect of the leading infrared renormalon has been canceled, and one expects the remaining perturbation series in (7) to be much better behaved than in the original expansion (6). Of course, one then needs an accurate measurement of $s_{1}$ or another kinematic moment. In Ref. [13], it is shown that the theoretical sensitivity of $s_{1}$ and $\left\langle E_{\ell}\right\rangle$ to $\bar{\Lambda}$ are roughly the same, once corrections of order $\left(\bar{\Lambda} / M_{B}\right)^{3}$ and necessary kinematic cuts are included. Either way, such an analysis is the strategy which will be required to reduce further the experimental error of $\left|V_{c b}\right|$.

\section{CHARMLESS INCLUSIVE SEMILEPTONIC $B$ DECAY}

I turn now to charmless semileptonic $B$ decay, and the inclusive width for $B \rightarrow X_{u} \ell \nu$. The partial decay rate $\Gamma\left(B \rightarrow X_{u} \ell \nu\right)$ is calculable as before, but unfortunately it is not very useful phenomenologically. The reason is that $\left|V_{u b}\right| /\left|V_{c b}\right| \sim 0.1$, so approximately $99 \%$ of semileptonic $B$ decays have charm in the final state. The only way to reject this huge background is by making kinematic cuts which exclude charmed final states unambiguously.

The simplest method experimentally, which has been used extensively in the past, is to require that the charged lepton in the decay have an energy larger than approximately

\footnotetext{
${ }^{\dagger}$ An explicit example of how this improvement works, up to order $\alpha_{s}^{5}$, is given in Ref. [12].
} 
$2.2 \mathrm{GeV}$, beyond the kinematic endpoint for decays to charm. This constitutes only a small tail of the spectrum $\mathrm{d} \Gamma / \mathrm{d} E_{\ell}$. In order to extrapolate to the complete partial width, it is necessary to have an accurate theoretical understanding of the shape of $\mathrm{d} \Gamma / \mathrm{d} E_{\ell}$ in the endpoint region. Unfortunately, while the OPE can be used to predict $\mathrm{d} \Gamma / \mathrm{d} E_{\ell}$ over most of the available phase space, it is actually an expansion in powers of $\bar{\Lambda} /\left(M_{B}-2 E_{\ell}\right)$ rather than $\bar{\Lambda} / M_{B}$ [8, 16]. In the endpoint region, where $E_{\ell} \simeq M_{B} / 2$, the OPE fails to converge reasonably and cannot be used for an accurate analysis.

As a result, the extraction of $\left|V_{u b}\right|$ by this method is extremely model dependent. Two approaches are used to address the issue. The first is inclusive, in which one performs the calculation as before but replaces the higher order terms in the OPE with a "shape function", which is an ansatz which resums formally an infinite set of terms in the series. This shape function is usually modeled by giving the $b$ quark a Gaussian momentum distribution [17]. The second is to treat the endpoint region as a sum over decays to individual exclusive final states, such as $\pi \ell \nu, \rho \ell \nu$, which are then analysed with a constituent quark potential model [18,19].

Each of these approaches has obvious deficiencies and misses important physics. Furthermore, even within each model there are uncertainties which cannot be quantified or even estimated, since these models are in no sense systematic approximations to QCD. There is an important moral to be drawn. In a situation such as this, analysing additional models does not lead to better accuracy in the determination of $\left|V_{u b}\right|$. Nor can the uncertainty in $\left|V_{u b}\right|$ due to the use of models be estimated sensibly simply by surveying the models currently available on the market. The model dependence in $\left|V_{u b}\right|$ is impossible to quantify reliably, and it continues to be underestimated consistently throughout the literature on this subject.

However, with the development of techniques for neutrino reconstruction, a new type of analysis has become possible. If the energy and momentum of the missing $\nu$ can be reconstructed kinematically, then it is possible to measure inclusively the invariant mass $s_{H}$ of the final state $X_{u}$. Charmed final states then can be rejected by requiring $s_{H}<\Delta^{2} \leq M_{D}^{2}$, where $\Delta^{2}$ is an experimental cut which may have to be less than $M_{D}^{2}$ because of details of the neutrino reconstruction procedure. The advantage of this approach is that most of the final states in $B \rightarrow X_{u} \ell \nu$ will pass such a cut, if $\Delta^{2}$ is reasonably close to $M_{D}^{2}$. One still must correct for the small "leakage" of rate to larger values of $s_{H}$, but, in contrast to the previous analysis, one is not extrapolating most of the rate from a small tail. Hence the larger $\Delta^{2}$ can be made to be, the smaller the effect of this tail on the measurement of $\left|V_{u b}\right|$, even if the tail is not modeled particularly well.

A careful analysis [20] (see also Ref. [21]) reveals that the fraction of rate with $s_{H}>\Delta^{2}$ depends crucially on the value of $\bar{\Lambda}$. This is true for two reasons. First, at tree level in the parton model, the spectrum $\mathrm{d} \Gamma / \mathrm{d} s_{H}$ cuts off at $s_{H}=\bar{\Lambda} m_{b}$, considerably below $M_{D}^{2}$. Thus, gluon bremmstrahlung plays an important role in creating states of large $s_{H}$, and this bremmstrahlung has a Sudakov-type double logarithmic singularity at $s_{H}=\bar{\Lambda} m_{b}$. The position of this (integrable) singularity controls the strength of the tail of the spectrum. Second, the nonperturbative corrections from the OPE have the effect of smearing the parton level spectrum by an amount of order $\bar{\Lambda} m_{b}$. When this smearing is modeled in the ACCMM model, it turns out that the dominant sensitivity of the size of the tail is to the value of $\bar{\Lambda}$; once $\bar{\Lambda}$ is fixed, the dependence of the leakage on other parameters, such as $\lambda_{1}$, is minimal.

Hence it is important, once again, to measure $\bar{\Lambda}$ using one of the inclusive methods 
discussed in the previous section. An accurate determination of this parameter is crucial for the accurate determination of $\left|V_{u b}\right|$. In addition, we should hope that the value of $\bar{\Lambda}$ turns out to be rather small, and that the experimenters can make $\Delta^{2}$ close to $M_{D}^{2}$, so that the separation between $\bar{\Lambda} m_{b}$ and $\Delta^{2}$ will be as large as possible. From the analysis of Ref. [20], one may estimate the eventual theoretical accuracy $\delta\left|V_{u b}\right|$ under a variety of hypothetical scenarios: $(i)$ if $\Delta^{2} \simeq M_{D}^{2}$ and $\bar{\Lambda}=400 \pm 100 \mathrm{MeV}$, then $\delta\left|V_{u b}\right| \sim 10 \%$; (ii) if $\Delta^{2} \simeq 1.5 \mathrm{GeV}^{2}$ and $\bar{\Lambda}=400 \pm 100 \mathrm{MeV}$, then $\delta\left|V_{u b}\right| \sim 30 \%$; (iii) if $\Delta^{2} \simeq 1.5 \mathrm{GeV}^{2}$ and $\bar{\Lambda}=200 \pm 100 \mathrm{MeV}$, then $\delta\left|V_{u b}\right| \sim 10 \%$; and $(i v)$ if $\Delta^{2} \simeq M_{D}^{2}$ and $\bar{\Lambda}=600 \pm 100 \mathrm{MeV}$, then $\delta\left|V_{u b}\right| \sim 50 \%$. With luck, this method eventually will yield the most accurate value of $\left|V_{u b}\right|$ available, a value largely free of model dependence.

\section{RADIATIVE $B$ DECAY}

Finally, I turn to the extraction of information about short distance dynamics from the inclusive radiative decay $B \rightarrow X_{s} \gamma$. Away from the region in which $M\left(X_{s}\right) \approx M(J / \psi)$, this decay is mediated primarily by a transition magnetic moment operator of the form $\bar{s} \sigma^{\mu \nu} F_{\mu \nu} b$. In the Standard Model, this operator is induced by one loop GIM-violating effects, and its coefficient is small. The total branching fraction for this decay in the Standard Model is at the level of $10^{-4}$. One the other hand, the coefficient of this operator, and hence the decay rate in this channel, is substantially larger in many models of new physics, especially those that postulate new flavor dynamics at the $\mathrm{TeV}$ scale. In order for an observed enhancement of $B \rightarrow X_{s} \gamma$ to serve as a signal of new physics, we must know how to predict the physical inclusive rate from a known quark operator such as $\bar{s} \sigma^{\mu \nu} F_{\mu \nu} b$.

In fact, the situation is quite analogous to that for $B \rightarrow X_{u} \ell \nu$, in that the total rate for $B \rightarrow X_{s} \gamma$ can be computed reliably with the OPE but is not quite useful phenomenologically 22,23 . Once again, the difficulty is the much larger number of decays to charmed final states. The most straightforward way to search for $B \rightarrow X_{s} \gamma$ is to look for the relatively hard $\gamma$, but there is an important background from the process $B \rightarrow D \pi^{0} \rightarrow D \gamma \gamma$, where one of the photons is missed. If one looks at the photon energy spectrum $\mathrm{d} \Gamma / \mathrm{d} E_{\gamma}$, it is unambiguously from the final state $X_{s} \gamma$ only for $E_{\gamma}>2.2 \mathrm{GeV}$. As a result, one is again in the position of extrapolating the bulk of the spectrum from a kinematic tail. For reasons similar to those which plague $B \rightarrow X_{u} \ell \nu$, the OPE is not convergent in the region to which one is restricted.

However, in some respects the situation is not quite as severe as for $B \rightarrow X_{u} \ell \nu$. At tree level in the parton model, the photon is monochromatic with $E_{\gamma} \simeq 2.4 \mathrm{GeV}$, above the kinematic cut. Radiative corrections and nonperturbative bound state effects smear the spectrum about this point, but most of the decay rate remains at large values of $E_{\gamma}$. In particular, even a relatively small downward shift in the value of $E_{\gamma}$ at which one must cut can reduce substantially the fraction of the rate which must be inferred by extrapolation. At present, however, the state of the art is still to use an inclusive model in this analysis, based on an Gaussian ansatz for the initial momentum distribution of the $b$ quark in the $B$ meson [24].

What is most important for the future is to aim for a measurement of $\mathrm{d} \Gamma / \mathrm{d} E_{\gamma}$ down to values of $E_{\gamma}$ as small as possible. Also, an accurate measurement of the shape of the spectrum 
in the region $E_{\gamma}>2.2 \mathrm{GeV}$ would help substantially to reduce the model dependence of the result, by providing information to constrain the models which are used. An accurate measurement of the spectrum in this region also could provide information on the shape function which governs the endpoint of the lepton energy spectrum in $B \rightarrow X_{u} \ell \nu$ [16,25], or could be used in an extraction of $\bar{\Lambda}$ and $\lambda_{1}$ which is complementary to that from the decay $B \rightarrow X_{c} \ell \nu$ [26,27].

\section{SUMMARY}

Inclusive $B$ decays are indispensable for the accurate extraction of $\left|V_{c b}\right|,\left|V_{u b}\right|$, and the coefficient of the operator responsible for the decay $b \rightarrow s \gamma$. While the theoretical treatment of such decays has developed into a mature field over the past five years, a number of subtleties remain. For the extraction of $\left|V_{c b}\right|$ from the dominant decay $B \rightarrow X_{c} \ell \nu$, the most important theoretical uncertainties are those in the inputs $\bar{\Lambda}$ (or $m_{b}$ ) and (to a lesser extent) $\lambda_{1}$, and from the truncation of the perturbative expansion at order $\alpha_{s}^{2}$. However, the infrared renormalon relates an ambiguity in the definition of $\bar{\Lambda}$ to an ambiguity in the resummation of the perturbative series, in such a way that the consistent elimination of $\bar{\Lambda}$ from physical quantities also helps to control the bad behavior of the perturbation series. Explicit strategies for extracting $\bar{\Lambda}$ from experiment are discussed.

For the rare decays $B \rightarrow X_{u} \ell \nu$ and $B \rightarrow X_{s} \gamma$, more serious theoretical uncertainties are induced by the severe kinematic cuts which must be imposed to eliminate the background from $B \rightarrow X_{c} \ell \nu$. A strategy for extracting $\left|V_{u b}\right|$ from $B \rightarrow X_{u} \ell \nu$, which relies on the experimental technique of neutrino reconstruction, has been developed. Future improvement in the theoretical understanding of inclusive semileptonic and rare $B$ decays will depend on (i) the direct measurement of $\bar{\Lambda}$ and $\lambda_{1}$ in inclusive decays themselves, a program which is already underway; and (ii) application of the loosest kinematic cuts possible in rare decays, which will help reduce the model dependence of the results. In the short and in the long term, the future in this field is bright; ultimately, the prospects are excellent for obtaining accurate and reliable insight into the CKM matrix and other short distance physics from inclusive semileptonic and radiative $B$ decays.

\section{ACKNOWLEDGMENTS}

I would like to thank the organizers for their gracious hospitality and a most stimulating meeting. This work was supported in part by the National Science Foundation under Grant No. PHY-9404057 and under National Young Investigator Award No. PHY-9457916, by the Department of Energy under Outstanding Junior Investigator Award No. DE-FG02-

94ER40869, and by the Alfred P. Sloan Foundation. A.F. is a Cottrell Scholar of the Research Corporation. 


\section{REFERENCES}

[1] A.F. Falk, M.B. Wise and I. Dunietz, Phys. Rev. D51, 1183 (1994).

[2] J. Chay, H. Georgi and B. Grinstein, Phys. Lett. B247, 399 (1990).

[3] I.I. Bigi, NlG. Uraltsev and A.I. Vainshtein, Phys. Lett. B293, 430 (1992);

I.I. Bigi et al., Phys. Rev. Lett. 71, 496 (1993).

[4] M. Beneke and V.M. Braun, Nucl. Phys. B426, 301 (1994);

M. Beneke, V.M. Braun and V.I. Zakharov, Phys. Rev. Lett. 73, 3058 (1994).

[5] A.F. Falk and M. Neubert, Phys. Rev. D47, 2965 (1994).

[6] M. Neubert and C.T. Sachrajda, Nucl. Phys. B438, 235 (1995).

[7] I.I. Bigi et al., Phys. Rev. D50, 2234 (1994).

[8] A.V. Manohar and M.B. Wise, Phys. Rev. D49, 1310 (1994).

[9] Y. Nir, Phys. Lett. B221, 184 (1989).

[10] M. Luke, M.J. Savage and M.B. Wise, Phys. Lett. B345, 301 (1995).

[11] A. Czarnecki and K. Melnikov, Nucl. Phys. B505, 65 (1997); Phys. Rev. Lett. 78, 3630 (1997).

[12] A.F. Falk, M. Luke and M.J. Savage, Phys. Rev. D53, 2491 (1996); D53, 6316 (1996).

[13] A.F. Falk and M. Luke, hep-ph/9708327, Phys. Rev. D57, to appear.

[14] M. Gremm et al., Phys. Rev. Lett. 77, 20 (1996);

M. Gremm and I. Stewart, Phys Rev. D55, 1226 (1997).

[15] M. Voloshin, Phys. Rev. D51, 4934 (1995).

[16] M. Neubert, Phys. Rev. D49, 3392 (1994); D49, 4623 (1994).

[17] G. Altarelli et al., Nucl. Phys. B208, 365 (1982).

[18] N. Isgur et al., Phys. Rev. D39, 799 (1989);

N. Isgur and D. Scora, Phys. Rev. D52, 2783 (1995).

[19] M. Baur, B. Stech and M. Wirbel, Z. Phys. C34, 103 (1987).

[20] A.F. Falk, Z. Ligeti and M.B. Wise, Phys. Lett. B406, 225 (1997).

[21] R.D. Dikeman and N.G. Uraltsev, hep-ph/9703437.

[22] A.F. Falk, M. Luke and M.J. Savage, Phys. Rev. D49, 3367 (1994).

[23] B. Blok et al., Phys. Rev. D49, 3356 (1994); D50, 3572(E) (1994).

[24] A. Ali and C. Greub, Phys. Lett. B361, 146 (1995); B293, 226 (1992).

[25] I.I. Bigi et al., Int. J. Mod. Phys. A9, 2467 (1994).

[26] A. Kapustin and Z. Ligeti, Phys. Lett. B328, 431 (1995).

[27] R.D. Dikeman et al., Int. J. Mod. Phys. A11, 571 (1996). 